\title{
The Lacquer Painting from Chinese Traditional Lacquer
}

\author{
Lina Dong \\ School of Design \\ Tianjin University of Commerce \\ Tianjin, China
}

\begin{abstract}
Since the sixth national art exhibition, lacquer painting was incorporated into art exhibition. This is a milestone of painting style's development, which becomes a more important form of artistic expression in today's China Art Circle. But the lacquer painting is still very young, it is always a decoration attached to the traditional lacquer with a rare cognition. In recent years the study of it limiting to materials and techniques, this article will research development course of lacquer painting from the archaeological literature.
\end{abstract}

\section{Keywords - tradition; lacquer; shape; lacquer painting}

\section{INTRODUCTION}

China, as a country of the largest amount of paint production and unearthing the most ancient lacquers, its lacquer is also world-famous, no matter in the designs, patterns, decorations full of elegance, which is known as the representative of the material culture of the Chinese nation with china and silk. The unique material technique of traditional Chinese lacquer art, is the precious heritage referenced and inherited by the modern lacquer painting. And the traditional lacquer coating also provides the rich basis for the birth and development of modern lacquer painting.

\section{AN HISTORICAL OVERVIEW OF CHINESE TRADITIONAL LACQUER}

The Chinese traditional lacquer ware was made of bamboo, wood, leather and linen, which appeared at early Shang. Warring States Han Feizi $\cdot$ Ten Faults recorded, "Yao gave the abdication to Yu Shun. As the feeder, cutting the wooden and sawing the shapes, painting liquid ink on it, was imported into the palace. Governors thought it's wasteful and kept a disagreement opinion. Then Shun gave the abdication to $\mathrm{Yu}$, who took it as a sacrificial ware with ink painting outside and red painting inside." As literature records, as early as in Yu Shun period, lacquer as appliances appeared. But lacquer crafts need spend more manpower and material resources with more tedious production. Governors thought it too extravagant and discourage $\mathrm{Yu}$ Shun. Then, Yu governed Hua Xia, took lacquer feeder as a sacrificial ware, which is the ware unearthed with ink paint outside and red paint inside. By the time of $\mathrm{Xia} \mathrm{Yu}$, the color of the lacquer was similar to the lacquer's and the common decoration of "ink outside and red inside". But it is more accurate to say that the lacquer-making process with wood as the bottom of the tire has appeared before $\mathrm{Yu}$ Shun and $\mathrm{Xia} \mathrm{Yu}$. For example, in 1978, the third cultural He Mudu ruins in Zhe Jiang, unearthed a lacquered wooden bowl, whose outside still remained a layer of scarlet coatings, after archaeological experts with infrared irradiation, spectrum after the analysis showing similarities with lacquer coating unearthed from Han Dynasty of Ma Wang. It was estimated that the site of hemudu site was about six or seven thousand years ago. It proves that the wooden bowl unearthed at the He Mu ruins in $\mathrm{Yu}$ Yao, Zhe Jiang province is the first piece of lacquer u to now..

In the Spring and Autumn period (770-476 BC), excavated lacquer objects: the lacquered box was unearthed in the 269th tomb of the Chang Zhi watershed in Shan Xi Province, which is decorated with painted curled-up dragon and Pan Chi, similar to contemporary bronzes. It is learned that, at that time, there was a certain decorative pattern on the lacquer, then the painter consciously and unconsciously had the adornment awareness.

The lacquer board unearthed from Northern Wei Dynasty's tomb of Sima Jinlong in Da Tong, Shan Xi province in 1966, with the theme of color drawing: fairy story and description of the image of tomb owner and his daily life. The wood paints are red, yellow, ocher, blue, green and white, which are very bright, with serious line drawing and observed composition. Compared with the same period of silk painting, in terms of the composition, the lines, they are pretty close, but different from decorative and patterning expressive lacquer painting in Han dynasty. Two complete pieces of lacquer screen unearthed from Sima Jinlong tomb, can be seen the bottom of wooden lacquer, whose technological process is painting vermilion lacquer on the board many times, then drawing by oil paint and mineral nature color paint. The subject is mainly about the character story pictures of the ancient filial sons and virtuous women.

A lacquer based on the painted figures was unearthed from Xi Han Dynasty's tomb from Hai Zhou distract, Lian Yungang city, Jiang Su province in 1973, with different and vivid characters. The lacquer body is painted in yellow, and the outline of the three men is in black lacquer. The head is all of plastic hair, tying the headscarf and wearing the right skirt, long sleeve robes. One is seemed to play music, one is 
dancing and the other is listening. There are decorative clouds among each of them, and the drawing technique is more innovative than before.

Another piece of the sacred monster with the tortoise shell was unearthed from the 8th Han tomb in Feng Huang Mountain, Hu Bei province at the same year, which is a valuable art. The surface of the painting shield is a shape of a turtle's belly, painted with black ochre paint. The shield is painted with a picture of a mysterious figure that looks like a human but not a human, the head and body are human characteristics, but the feet are animals', wearing leopard striped trousers, and another raising head and bending body, like a flying beast. The image on the back of the shield is the opposite, wearing the same costume, and the man on the right has a long sword. In composition, the artist has a relatively symmetrical composition according to the shape of the turtle's shield, outlining the characters and beasts, and the painting with a simple and cursory line, which increases the power of the divine beast. The painted shield images of both the beasts and characters are a few lines, but the style is more advanced, the picture seeming simple but vivid, and coinciding with the modern style.

\section{THE TRANSFORMATION FROM CHINESE TRADITIONAL LACQUER TO GRAPHIC LACQUER PAINTING}

From the unearthed historical relics, whether from the decoration or a pure form, early lacquers have shown the shadow of the lacquer painting, which is a part of the lacquer, as an art sketch appeared to fill in the void space. As far as the lacquer art is concerned, the paint is mostly planar, abstract and decorative, and also has realistic painting scene, which depicts the external shape of the contents around the shape of the utensil. The lacquer has bright hues, vivid decorative patterns, dynamic and rhythmic lines, which show the sense of order in pause and transition. The lacquer is full of simple tone, dignified and primitive; the composition between the midpoint and line of pattern, just like abstract painting at this time, which is both decorative and modern. In artistic expression, compared with contemporary painting works, the difference is just in the expression technique.

\section{A. The Change of Pattern}

The modern painting, derived from the seven thousand years of lacquer tradition, is the same as the Chinese painting and oil painting, which is named after the material. In the construction of contemporary urban culture, traditional lacquer materials and technology are consciously transforming from the traditional form to the modern. There is the necessity of the lacquer art developing to modern lacquer painting. In the modern society of environment determining culture, paying attention to the aesthetic needs, the artists inject traditional culture into the modern spirit and consciousness, which makes the lacquer art close to real life, not divorcing from modern people's thoughts and feelings. If it is divorced from the reality, it can only be a pure handicraft, not an independent art form. Modern lacquer art gradually has a trend of getting away from the needs of material life, just to be an opposite object of pure aesthetic, which is not only another drawback in the development of lacquer art, but also greatly reduces the development of lacquer art space. We can imagine that "practicality is beauty", only strengthening the research and practice of its practicality will make the traditional process more vitality, and more room for development.

Such as Japan's modern lacquer striving to close to the market and public life on the design orientation, people cannot leave the lacquer in building decoration, display art, furniture and gifts design and catering exposure daily, which has risen as a part of Japanese life. So, compared with Japan, the Chinese contemporary lacquer art in the public environment decoration development has a very big space, determined by its characteristics. Lacquer art full of plasticity, various skills, anticorrosive, is an ideal indoor and outdoor decorative materials. This art technology can be combined with sculpture, not only for wall decoration, can also be used to the design of screen, wall hanging, furniture, to create the human's ideal environment.

\section{B. Renewal of Materials}

Natural lacquer has a cool and rich tone, beautiful and implicit color, while synthetic chemical painting color shows a clear and translucent aesthetic feeling. It makes lacquer painting more room to develop that new materials are emerging, techniques and styles are different and the traditional techniques are continuously modified with a creative use of new paint. For example, cashew paint and synthetic paint can be naturally dry without depending on the shade, and pigments are more abundant than before, and allergies and toxicity are decreased. And with the help of advantages of modern synthetic paint, the lacquer painting gradually enter into art forms, such as the murals, sculptures, public art, indoor display, to promote its social influence, widen its visual expression. Chinese lacquer painting creation reflects the unity of traditional culture and modern innovative, rich and deep connotation of traditional lacquer art, independent aesthetic style constituting traditional lacquer painting. It combines painting and technology, focusing on the richness of artistic expression and the wide adaptability of subject, and trying to innovate the artistic conception of shape art and culture connotation.

\section{Independence of Lacquer Painting}

Nowadays, the reason why lacquer painting has come a long way in the contemporary art world is that its unique charm material, which comes from the characteristics of the paint itself. As the traditional Chinese painting taking ink, paper as raw materials, oil painting taking paint and canvas as raw materials, lacquer painting is constructed on the basis of the main media materials of lacquer or synthetic lacquer. Lacquer painting is the crystallization of two traditional inheritors of the materials' technique and Chinese modern painting. As Gombrich put it, "In ancient China, there was no way to go beyond the desire of others. In China, it is better to inherit than to break."

Contemporary lacquer painting is no longer the accessory of the lacquer, but as an independent painting kind from the mother of ancient Chinese lacquer. And it is more artistic, going to the plane, to the pure abstract shape, and then seeks 
for change with the time changing. And the lacquer that is left behind, has got away from the aristocratic life, from the object owned only a few people to going up to antique shelf of modern thousands of family.

\section{CONCLUSION}

In the end, the lacquer painting is out of the mother of Chinese traditional lacquer, going to the plane, to the pure abstract shape. And constantly changing with The Times, seeking for change and making it more contemporary, lacquer painting has been brought into the national art exhibition since the 6th national art exhibition. For lacquer painting itself, this is a milestone in the development of painting style. However, compared with other paintings, lacquer paintings are still relatively young, since lacquer paintings have always taken traditional techniques as the main content. Therefore, in the process of development, lacquer painting should always refer to the expression of other paintings, combine with the current spiritual needs and the renewal of values, and constantly enhance the nature of lacquer painting.

\section{REFERENCES}

[1] Hemudu Site Investigation Group: The Major Gain Explored the Second in Hemudu Site in Zhe Jiang, Historical Relic. 1980, the fifth phrase

[2] Li Zhengguang editing: The Design of Lacquer in Han Dynasty, Cultural Relics Press, 2002,Dec publishing, the second page

[3] Wang Shixiang: The Chinese Ancient Lacquer, Cultural Relics Press, 1987

[4] Zhu Gekai: Ink and Red Paint: The Chinese Lacquer Art, Bei Jing,SDX, Joint publishing Company, 2000 June edition the 79th page

[5] Lu Jie: On the Decoration Craft and Development Value of Lacquer in Han Dynasty, Master's Thesis of Nan Jin Normal University, 2005,6 . 